\title{
Impulsive Taxation of Diabetic Maculopathy from Tint Retinal Metaphors
}

\author{
R. Sukanesh,M.E, Ph.D \\ Department of ECE, \\ Thiagarajar College of Engineering, \\ Madurai, Tamil Nadu, India
}

\author{
S. MURUGESWARI, \\ Department of ECE, \\ Syed Ammal Engineering College \\ Ramanathapuram, Tamil Nadu, India
}

\begin{abstract}
Diabetic Maculopathy (DM) is a foremost cause of blindness. Exudates are one of the crucial signs of diabetic maculopathy which is a main cause of blindness that could be prevented with an early screening process. In this approach, the process and consciousness of digital image processing to diagnose exudates from images of retina is applied. Presence of exudates and Maculopathy is focused from low-contrast digital images of Diabetic patients' with non-dilated pupils is proposed. Image is segmented by using colour K-means Clustering algorithm. Then segmented image along with Optic Disc (OD) is chosen. Next segmented region, features and texture are extracted. The nominated feature vector are then classified into exudates and non-exudates using a Support Vector Machine (SVM) Classifier. Diabetic Maculopathy, which is the severe stage of Diabetic Retinopathy is accomplished using Morphological Operation. This method performs auspicious as it can detect the very small areas of exudates. Enforced mass airing will help to identify the maculopathy at early stage and reduce the risk of unembellished vision loss. Diabetic Maculopathy is sensed with $100 \%$ success rate.
\end{abstract}

\section{Keywords}

Diabetic Maculopathy, Fuzzy k-Means, Exudates, Dilated Retinal Images.

\section{INTRODUCTION}

DM is generally detected directly or indirectly. Direct ways are using stereoscopy (for manual examination) or optical computed tomography images [3]. Indirect method is by detecting the presence of hard exudates (HE) in the retina. HE are formed due to secretion from capillaries resulting from the complications of retinal vasculature and could lead to retinal swelling.

In color fundus images they appear as yellow-white deposits (see figure 3a). Detecting the presence of hard exudates (HE) in different areas of retina is now considered a standard method to assess DM from tint fundus images [1], [4], [5].

The brutality of the jeopardy is assessed based on the vicinity of $\mathrm{HE}$ to the macula, which is defined to be a circular region centered at fovea and with 1 optic disc (OD) diameter (see figure 1). The jeopardy for $\mathrm{DM}$ increases when the $\mathrm{HE}$ locations approach the macula, with the jeopardy being the highest, when they are within the macula.

Macula is a perceptive region in the central part of the human eye retina, conscientious for high clarity vision, color vision and central vision. There are different conditions that damage the macula, so that the central part of the image becomes blurred, only the peripheral parts remaining clear. Macular diseases, are an increasing problem worldwide, because they are irreversible, so it is very important that the process to be early identified and stopped. Macular degeneration is caused by the deterioration of the central portion of the retina,back layer of the eye that records images and sends them via the optic nerve from the eye to the brain. Such problems may occur at a higher age, but in the last years, they were observed at the second part of peoples' life $(40-60$ years old). It has a diameter of around $1.5 \mathrm{~mm}$ and having two or more layers of ganglion cells.

Near its center is the fovea, a small pit that contains the largest concentration of cone cells in the eye and is answerable for central, high resolution vision. The macula also comprises the Para fovea and per fovea. The macula is yellow in colour it absorbs excess blue and ultraviolet light that enters the eye, and acts as a natural sunblock (analogous to sunglasses). The lutein and zeaxanthin jointly give the yellow color for the macula, derived from the diet. Then, these carotenoids protect the pigmented region from some types of macular degeneration. The macula is specialized for high clarity vision. Within the macula are the fovea and favela which contain a high density of cones (photoreceptors with high acuity).

Whereas loss of peripheral vision may damage the macula and will loose central vision. The enlightened demolition of the macula is a disease known as macular degeneration and can sometimes lead to the creation of a macular hole. Macular holes are rarely caused by distress, but if a severe blow is delivered it can burst the blood vessels going to the macula and destroying it. Visual input from the macula occupies a considerable portion of the brain's visual capacity.

Diabetes can also cause other retinal snags all of which are communally termed as diabetic retinopathy (DR).Given the potential for vision loss and blindness due to DR ,screening programs have been launched in many countries and tint fundus image forms the basis for manual assessment in screening. Such manual assessment however is not climbable in large-scale screening scenario, particularly in developing countries either due to the scarceness of skilled manpower or inapproachability of high end imaging equipment at the point of care. Solutions such as tele-screening using enduring and mobile units to enable airing of retinal disorders in remote areas have been proposed[6] and [7]. 


\section{PROPOSED METHOD FOR THE DETECTION OF EXUDATES IN COLOUR FUNDUS}

\subsection{State of Art}

Alireza Osareh et al [4] proposed a method for automatic identification of exudates based on computational Intelligence technique The colour retinal images were segmented using fuzzy c-means clustering. Feature vector were extracted and classified using multilayer neural network classifier.

Akara Sopharak et al [5] reported the result of an automated detection of exudates from low contrast digital images of retinopathy patients with non-dilated pupils by Fuzzy CMeans clustering. Four features applied as input to coarse segmentation using FCM clustering method. The detected result were validated with expert ophthalmologists Sensitivity, Specificity, positive predictive value (PPV), positive likelihood ratio (PLR) and accuracy were used to assess the overall enactment of the system.

Niemeijer et al [6] distinguished the bright lesion like exudates, cotton wool spots and drusen from colour retinal images. First pixels were classified with probability map that included the probability of each pixel to be part of a bright lesion. Then, pixels with high probability were grouped into probable lesion pixel clusters. Based on cluster, each cluster was assigned a probability indicating that the cluster was a true bright lesion. Lastly these clusters were classified as exudates, cotton wool spots or drusen. Sensitivities and specificities of the annotations on the 300 images by the automated system were obtained.

Akara sopharak et al [7] proposed a series of experiments on feature selection and exudates classification using naive bayes and Support Vector Machine (SVM) Classifiers. At first, they used naive bayes model to a training set consisting of 15 features extracted from positive and negative examples of exudates pixels. To obtain the best SVM, they used the best feature set from the naive bayes classifier and recurrently attached the removed features to the classifier They carried out a grid search to find the best combination of hyper parameters like tolerance for training error and radial basis function width by taking each combination of features. They compared the best naive bayes and SVM classifier to a Nearest Neighbors classifier. They proved that the naive bayes and SVM classifiers executed better than the NN classifier.

Walter et al [8] identified exudates from green channel of the retinal images according to their gray level variation. Mathematical morphology techniques were used to determine the exudates contour. This method used three parameters: size of the local window and two threshold value.

Exudates regions were initially found using first threshold value. The second threshold represents the minimum value, from which a candidate pixel must differ from its surrounding background to be classified as exudates. The author achieved a sensitivity of $92.8 \%$ and predictivity of $92.4 \%$ against a set of 15 abnormal retinal images. However the author ignored some types of errors on the border of the segmented exudates in their reported performances and did not discriminate exudates from cotton wool spots.

Sinthanayothin et al [9] reported the result of an automated detection of Diabetic retinopathy by Recursive Region Growing techniques on a $10 \times 10$ window using selected threshold value. In the pre-processing steps, adaptive local contrast enhancement is applied. The author reported a sensitivity of $88.5 \%$ and specificity of $99.7 \%$ for the detection of exudates against a small dataset comprising 21 abnormal and 9 normal retinal images.

Phillips et al [10] identified the exudates by using Global and local thresholding. The input images were pre-processed to eliminate photographic non-uniformities and the contrast of the exudates was enhanced. Based on this technique sensitivity was reported between $61 \%$ and $100 \%$ by using 14 images. A weakness of this method was that other bright lesions (such as cotton wool spots) could be identified mistakenly.

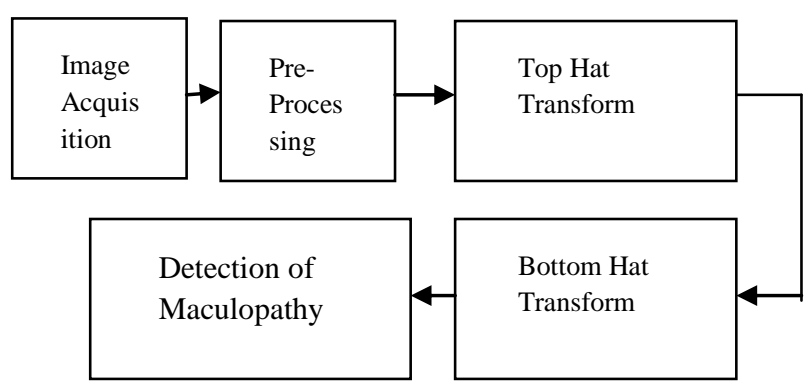

Fig. 2. Block Diagram of Proposed Method

\subsection{Image Acquisition:}

To estimate the enactment of this technique, the digital retinal images were acquired using Topcon TRC-50 EX camera with a $50^{\circ}$ field of view at Rajan's Eye Hospital ,Chennai.

\subsection{Pre-Processing}

Colour fundus images often indicate the lighting variation, poor contrast and noise. To reduce these imperfection [11] and generate images more suitable for extracting the pixel features by classification process. In pre-processing comprising the following step is applied. 1) RGB to HSI conversion 2) Median Filtering 3) Contrast Limited Adaptive Histogram Equalization (CLAHE).

\subsubsection{Extracting the Green Component}

Since the green component contains more information, it is extracted from the input retinal images in RGB Colour space. The noise in the images are due to the uneven distribution of the intensity(I) component.

\subsubsection{Median Filtering:}

In order to uniformly distribute the intensity throughout the image, the I-component of HSI color space is extracted and filtered out through a $3 \mathrm{X} 3$ median filter.

\subsubsection{Contrast Limited Adaptive Histogram Equalization (CLAHE):}

An adaptive histogram equalization is applied on the filtered I-component of the image [12].

\section{DETECTION OF MACULOPATHY \\ 3.1 Erosion:}

The erosion of the binary image $A$ is defined by the structuring element $B$ is

$$
A \ominus B=\left\{z \in E \mid B_{z} \subseteq A\right\},
$$


the translation of $B$ by the vector $z$ is denoted by $B_{Z}$,

$$
B_{\approx}=\{b+z \mid b \in B\}, \forall z \in E \text {. }
$$

When the structuring aspect B has a center (e.g., B is a disk or a square), and the center is located on the foundation of $\mathrm{E}$. Then the erosion of A by B can be implicited as the locus of point attained by the center of $\mathrm{B}$, when $\mathrm{B}$ moves inside $\mathrm{A}$. let the erosion of a square of side be 10 , centered at the foundation, by a disc of radius 2 , also centered at the foundation, is a square of side 6 centered at the foundation. The erosion of A by B is expressed as:

$$
A \ominus B=\bigcap_{b \in B} A_{-b} \text {. }
$$

\subsection{Dilation:}

The dilation of the dark-blue square from the disk, resulting in the light-blue square with rounded corners.

The dilation of $A$ from the structuring element $B$ is defined by:

$$
A \oplus B=\bigcup_{b \in B} A_{b} .
$$

The dilation is commutative, also given by:

$$
A \oplus B=B \oplus A=\bigcup_{a \in A} B_{a}
$$

If $B$ has a center on the foundation, as before, then the dilation of $A$ by $B$ can be known as the locus of the points enclosed by $B$, when the center of $B$ moves inside $A$.

The dilation can also be obtained by:

$A \oplus B=\left\{z \in E \mid\left(B^{s}\right)_{\approx} \cap A \neq \varnothing\right\}$, where $B^{s}$ is the sectional of $B$, that is

$$
B^{s}=\{x \in E \mid-x \in B\} \text {. }
$$

\subsection{Proposed Algorithm:}

i. Get the input image.

ii. Obtain the Green Component of the image (G) from the original RGB image.

iii. Apply median filter and adaptive histogram equalization to the Green component of the image.

iv. Apply Top-Hat transform to the image $G$ and add the result with the image $\mathrm{G}$. Then the output image is $\mathrm{T} 1$.

v. Apply Bottom-Hat transform to the image G.

vi. Subtract the result with the image T1.

vii. Macula, which is the darkest region of an image is detected.

viii. End the process

\section{RESULT AND DISCUSSION}

Macula is the darkest part in the retinal image. Macula is localized using morphological operation. If exudates is presented in macula region, then it will indicate the presence of Diabetic Maculopathy.
If exudates is not present in this region, then it shows the absence of Diabetic Maculopathy.

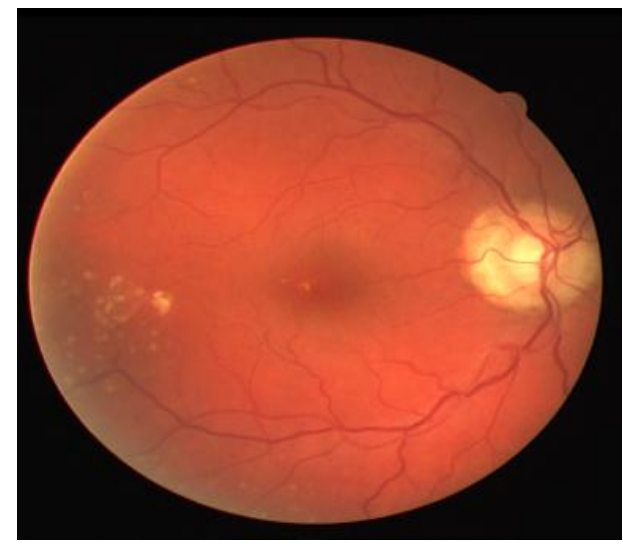

Fig .3 a) Colour fundus Image

Figure 3.a shows the colour fundus image affected with Diabetic Maculopathy. The centre darkest region is the macula. In this approach, the Diabetic Maculopathy is detected using morphological operation and it is indicated in Figure 3.b using the rectangular box.

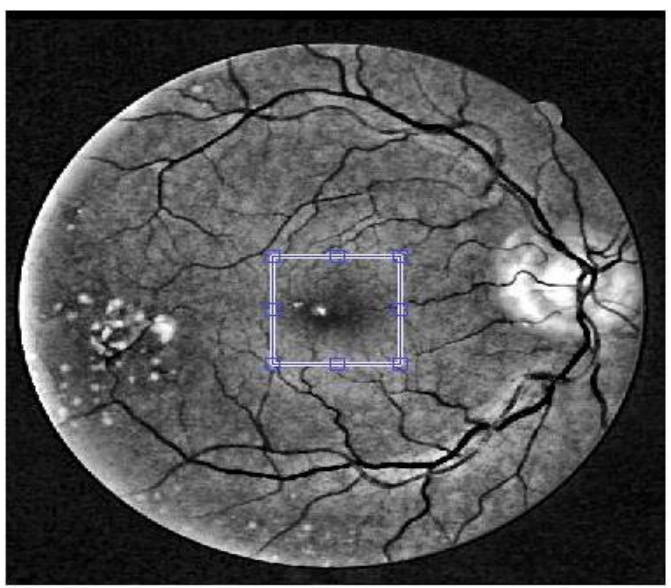

Fig .3 b) Detection of maculopathy

The diabetic retinopathy images were collected from STARE and DRIVE database. Exudates are one of the earlier sign of diabetic Maculopathy. The low contrast digital image is enhanced by using an Adaptive Histogram Equalization (CLAHE). The noise are removed from the images using median filter. Diabetic Maculopathy, which is the severe stage of Diabetic Retinopathy is detected using morphological operation. The method is evaluated on 70 abnormal and 30 normal images. Out of 100 images, 100 images were detected successfully and thus a success rate of $100 \%$ was obtained . 
Table- I Comparision of our Proposed Method with Existing Method

\begin{tabular}{|l|l|l|l|}
\hline S.No & Classifier & $\begin{array}{l}\text { Success Rate } \\
(\%)\end{array}$ & $\begin{array}{l}\text { Time to } \\
\text { Execute } \\
\text { (Approx) }\end{array}$ \\
\hline 1 & k-NN Classifier & 92 & $4.2 \mathrm{~min}$ \\
\hline 2 & SVM Classifier & 96 & $2.3 \mathrm{~min}$ \\
\hline 3 & Navie Baye's & 85 & $1.5 \mathrm{~min}$ \\
\hline 4 & Neural Network & 90 & $3.5 \mathrm{~min}$ \\
\hline 5 & $\begin{array}{l}\text { Fuzzy k-means } \\
\text { Classifier }\end{array}$ & 92 & $2.4 \mathrm{~min}$ \\
\hline 6 & Our Method & 100 & $25 \mathrm{sec}$ \\
\hline
\end{tabular}

\section{REFERENCES}

[1] Olson. J. A, Strachana. F.M, Hipwell. J. H, “A comparative evaluation of digital imaging, retinal photography and optometrist examination in screening for diabetic retinopathy" Journal on Diabet Med. Vol. 20, No. 7 .pp. 528- 534, July 2003.

[2] Luca Giancardo*, Student Member, IEEE, Fabrice Meriaudeau, Member, IEEE,. “ Textureless Macula Swelling Detection With Multiple Retinal Fundus Images" IEEE TRANSACTIONS ON BIOMEDICAL ENGINEERING, VOL. 58, NO. 3, MARCH 2011

[3] Alireza Osareh, Bita shadgar and Richard Markham,“A computational intelligence based approach for detection of exudates in Diabetic Retinopathy Images", IEEE Trans. on Information Technology in Biomedicines, vol.13, no. 4, pp. 535-545, July 2009.

[4] Alireza Osareh, Bita shadgar and Richard arkham,"A computational intelligence based approach for detection of exudates in Diabetic Retinopathy Images", IEEE Trans. on Information Technology in Biomedicines, vol. 13, no. 4, pp. 535-545, July 2009.

[5] Akara Sopharak, Bunyarit Uyyanonvara, Sarah Barman, "Automatic Exudate Detection from Non-dilated Diabetic Retinopathy retinal images using Fuzzy CMeans Clustering" Journal of Sensors, vol.9, No. 3, pp 2148- 2161, March 2009

[6] Niemeijer. B.V, Ginnekan. S. R, Russell. M, and M.D. Abramoff, "Automated detection and differentiation of drusen, exudates and cotton- wool spots in digital color fundus photographs for diabetic retinopathy diagnosis", Journal on Investigate Ophthalmol. And Visual Science., vol. 48, No. 2 pp. 2260-2267, 2007.

[7] Akara Sopharak, Mathew N. Dailey, Bunyarit Uyyanonvara, Sarah Barman, Tom Williamson, Yin Aye Moe, "Machine Learning approach to automatic Exudates detection in retinal images from diabetic patients", Journal of Modern optics, Vol. 57, No. 2, pp. 124-135, Nov 2011

[8] T. Walter, J.Klein, P.Massin and A.Erginary, "A Contribution of image processing to the diagnosis of Diabetic Retinopathy detection of exudates in color fundus images of the human retina", IEEE Trans. On Med. images, vol. 21, no. 10, pp. 1236-1243, 2002.

[9] C. Sinthanayothin, "Image analysis for automatic diagnosis of Diabetic Retinopathy", Journal of Medical Science, Vol. 35,No. 5, pp. 1491-1501, Jan 2011.
[10] Fleming. AD, Philips. S, Goatman. KA, Williams. GJ, Olson. JA, sharp. PF, "Automated detection of exudates for Diabetic Retinopathy Screening", Journal of Phys. Med. Bio., vol. 52, no. 24, pp. 7385-7396, 2007.

[11] Guoliang Fang, Nan Yang, Huchuan Lu and Kaisong Li, "Automatic Segmentation of Hard Exudates in fundus images based on Boosted Soft Segmentation", International Conference on Intelligent Control and Information Processing, pp. 633-638, Sept 2010.

[12] Pizer. S.M. "The Medical Image Display and analysis group at the university of NorthCarolina:Reminiscences and philosophy" IEEE Trans On Medical Imaging, vol. 22, no. 1, pp. 2-10, April 2003.

[13] Plissiti.M.E., Nikar.C, Charchanti.A, "Automateddetection of cell nuclei in pap smear images using morphological reconstruction and clustering" IEEE Trans. On Information Technology in Biomedicine, vol.15,no. 2, pp. 233-241, March 2011

[14] Seongijin park, Bohyoung Kim, Jeongjin Loe“ GGO nodule volume preserving Non-rigid Lung Registration using GLCM texture analysis", IEEE Trans. On Biomedical Engg., vol. 58, no. 10, pp. 2885-2894, sept 2011.

[15] Kandaswamy.U, Adjerch.D.A, Lee.M.C, "Efficient Texture analysis of SAR imagery", IEEE Trans. On Geoscience and Remote Sensing, vol. 43, no. 9,pp. 2075-2083, August 2005.

[16] Tobin.K.N, Chaum.E, Govindasamy.V.P, "Detection of anatomic structures in human retinal imagery" IEEE Transactions on medical imaging, vol. 26, no. 12,pp. 1729-1739, December 2007.

[17] Gwenole Quellec, Stephen R. Russell, and Michael D. Abramoff, Senior Member, IEEE "Optimal Filter Framework for Automated, Instantaneous Detection of Lesions in Retinal Images" IEEE Trans. on medical imaging, vol. 30, no. 2,pp. 523-533, February 2011.

[18] Akara Sopharak, Bunyarit Uyyanonvara, sarah Barman, "Comparative analysis of automatic exudates detection algorithms", Proceedings of the world congress onEngg., Vol I, Dec 2011.

[19] Doaa Youssef, Nahed Solouma, Amr El-dib, Mai Mabrouk, "New Feature-Based Detection of Blood Vessels and Exudates in Color Fundus Images" IEEE conference on Image Processing Theory, Tools and Applications,2010,vol.16,pp.294-299.

[20] Seongijin park, Bohyoung Kim, Jeongjin Loe“ GGO nodule volume preserving Non-rigid Lung Registration using GLCM texture analysis", IEEE Trans. On Biomedical Engg., vol. 58, no. 10, pp. 2885-2894, sept 2011.

[21] Kandaswamy.U, Adjerch.D.A, Lee.M.C, "Efficient Texture analysis of SAR imagery", IEEE Trans.

On Geoscience and Remote Sensing, vol. 43, no 9,pp. 2075-2083, August 2005.

[22] Tobin.K.N, Chaum.E, Govindasamy.V.P, "Detection of anatomic structures in human retinal imagery" IEEE Transactions on medical imaging, vol. 26, no. 12,pp. 1729-1739, December 2007. 
[23] Gwenole Quellec, Stephen R. Russell, and Michael D. Abramoff, Senior Member, IEEE "Optimal Filter Framework for Automated, Instantaneous Detection of Lesions in Retinal Images" IEEE Trans. on medical imaging, vol. 30, no. 2,pp. 523-533, February 2011.

[24] Akara Sopharak, Bunyarit Uyyanonvara, sarah Barman, "Comparative analysis of automatic exudates detection algorithms", Proceedings of the world congress on Engg., Vol I, Dec 2011.

[25] Doaa Youssef, Nahed Solouma, Amr El-dib, Mai Mabrouk, "New Feature-Based Detection of Blood Vessels and Exudates in Color Fundus Images“ IEEE conference on Image Processing Theory, Tools and Applications,2010,vol.16,pp.294-299.

[26] Laszlo Kovacs, Rashid Jalal Qureshi, Brigitta NagyBalazs Harangi, Andras Hajdu "Graph Based Detection of Optic Disc and Fovea in Retinal Images" Faculty of Informatics, University of Debrecen, POB 12, 4010 Debrecen, Hungary

[27] [21] Ziyang Liang, Damon W.K. Wong, Jiang Liu, Kap Luk Chan, Tien Yin Wong "Towards automatic Detection of age-related macular degeneration in retinal fundus images" 32nd Annual International Conference of the IEEE EMBSBuenos Aires, Argentina August 31 September 4, 2010

[28] Thomas Walter3, Jean-Claude Klein, Pascale Massin, and Ali Erginay “ A Contribution of Image Processing to the Diagnosis of Diabetic Retinopathy-Detection of Exudates In Color Fundus Images of the Human Retina" IEEETransactions on medical imaging, vol. 21, NO. 10, October 2002.

[29] Harihar Narasimha-Iyer, Ali Can, Badrinat Roysam and Jeffrey Stern "Automated Change Analysis From
Fluorescein Angiograms for Monitoring Wet Macular Degeneration" Proceedings of the 28th IEEE EMBS Annual International Conference New York City,USA,Aug 30-Sept 3, 2006.

[30] Kenneth W. Tobin*, Senior Member, IEEE, Edward Chaum, V. Priya Govindasamy, Member, IEEE, and Thomas P. Karnowski, Member, IEEE "Detection of Anatomic Structures inHuman Retinal Imagery" IEEE transactions on medical imaging, vol. 26, no. 12, december 2007.

[31] Mona K. Garvin*, Member, IEEE, Michael D. Abràmoff,Member, IEEE, Randy Kardon, Stephen R. Russell,Xiaodong Wu, Senior Member, IEEE, and Milan Sonka, Fellow, IEEE"Intraretinal Layer Segmentation of Macular OpticalCoherence Tomography Images Using Optimal3-D Graph Search “ IEEE Transactions On Medical Imaging, Vol. 27, No. 10, October 2008.

[32] Doaa Youssef1, Nahed Solouma1, Amr El-dib1, Mai Mabrouk2,andAbo-BakrYoussef3"New Feature-Based Detection of Blood Vessels and Exudates in Color Fundus ImageImage ProcessingTheory, Tools and Applications.

[33] Gwénolé Quellec*, Kyungmoo Lee, Student Member, IEEE, Martin Dolejsi, Mona K. Garvin, Member, IEEE "Three-Dimensional Analysis of Retinal LayerTexture: Identification of Fluid-FilledRegions in SD-OCT of the Macula"IEEE Transactions On Medical Imaging, Vol. 29, No. 6, June 2010

[34] M. Luculescu, S. Lache, D. Barbu and I. Barbu, "Feature extraction methods used for images of macular diseases - Part II", The 1st Interna-tional Conference on Computational Mechanics and Virtual Engineering COMEC 2005, Braşov, Vol. II, 2005, pp. 55-60.

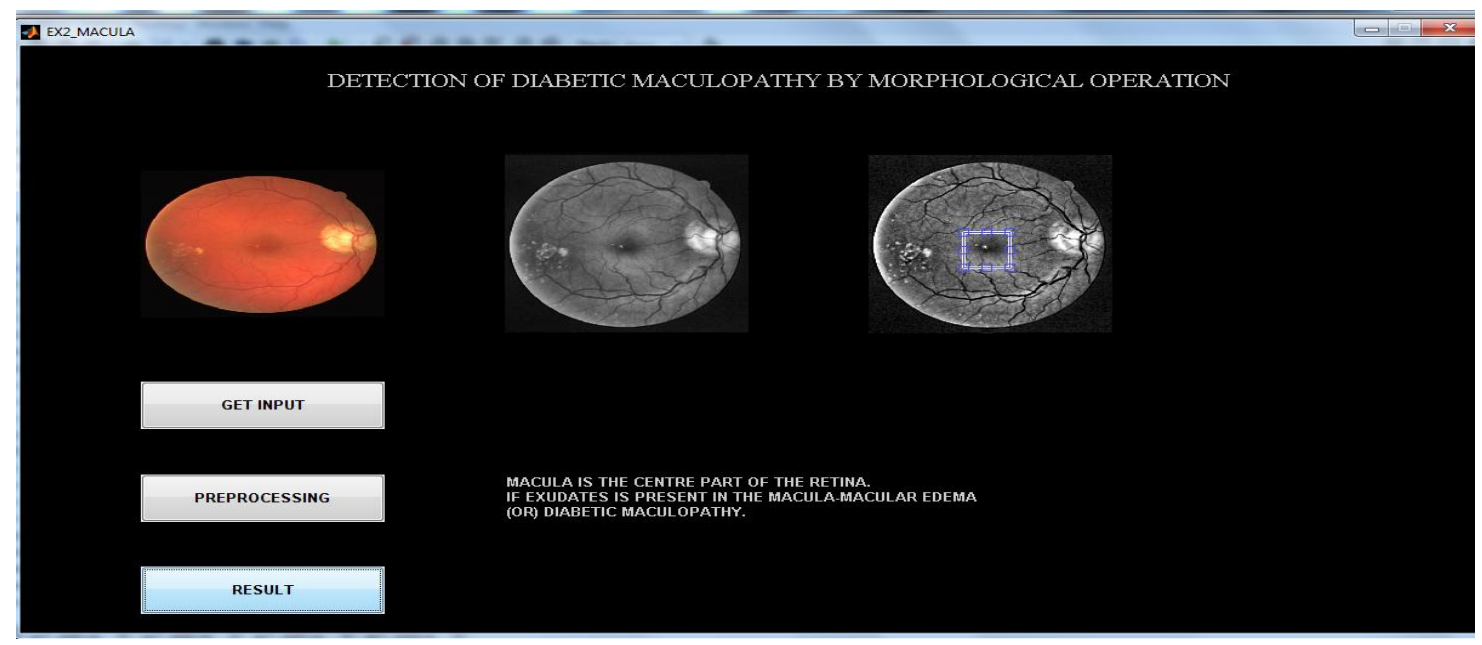

Fig. 3 c) Detection of Diabetic Maculopathy in a GUI window 